\title{
ANÁLISE RADIOGRÁFICA DO TRATAMENTO CIRÚRGICO DA FRATURA CERVICAL BAIXA POR VIA POSTERIOR
}

\author{
RADIOGRAPHIC EVALUATION OF THE POSTERIOR FIXATION IN THE SUBAXIAL \\ CERVICAL SPINE INJURY

\section{EVALUACIÓN RADIOGRÁFICA DE LA FIJACIÓN POSTERIOR DE LA FRACTURA DE LA COLUMNA CERVICAL BAJA}

Nelson Astur Neto', Luciano Antonio Nassar Pellegrino², Ricardo Shigueaki Galhego Umeta², Maria Fernanda Silber Caffaro³, Robert Meves ${ }^{4}$, Elcio Landim ${ }^{5}$, Osmar Avanzi ${ }^{6}$

\section{RESUMO}

Objetivo: Avaliação da evolução radiográfica da lesão da coluna vertebral cervical tratada pela fixação posterior isolada. Métodos: De 2000 a 2008 foram selecionados 23 pacientes que atenderam aos critérios de inclusão do estudo. Eram masculinos 91,3\% e a idade média foi de 34 anos e quatro meses. O tempo de seguimento médio foi de 82 meses. Foi avaliado no exame de imagem pré-operatório, pós-operatório imediato e após seis meses de evolução o tipo de implante, a consolidação da artrodese, se houve soltura do implante, perda de redução, cifose segmentar, degeneração de nível adjacente e pseudartrose. Resultados: Em relação ao método de síntese, $60,8 \%$ dos pacientes foram submetidos ao amarrilho interespinhoso, 26\% à placa com parafusos de massa lateral e $13 \%$ à barra com parafusos de massa lateral. Dos pacientes submetidos à fixação com parafusos, nenhum apresentou complicações radiográficas e $35,7 \%$ dos pacientes submetidos à artrodese com amarrilho interespinhoso tiveram complicação, sendo a mais frequente a cifose segmentar. Conclusão: As lesões da coluna cervical submetidas a artrodese com parafuso de massa lateral apresentaram uma evolução radiográfica melhor do que as submetidas a fixação com amarrilho interespinhoso, tendo este último apresentado maior incidência de complicações na artrodese.

Descritores: Vértebras cervicais; Fixação de fratura; Dispositivos de fixação ortopédica; Fixadores internos; Fraturas da coluna vertebral; Traumatismos da coluna vertebral; Cerclagem cervical.

\begin{abstract}
Objective: To perform a radiographic evaluation of the cervical spine injury treated with posterior fixation techniques only. Methods: From 2000 to 2008, twenty three patients were included in the study, of which 91,3\% were men, with a mean age of thirty-four years and four months. The mean follow-up time was 82 months. The type of implant used, the radiographic arthrodesis consolidation, implant failure, lost of reduction, segmental kyphosis and pseudarthrosis were evaluatedin the preoperative period, the immediate postoperative period and after six months of evolution, based on the patients records. Results: When it comes to the type of implant used, there were 60,8\% of the patients who underwent interspinous wire fixation, $26 \%$ with lateral mass screws and plate and $13 \%$ with lateral mass screws and rods. Of the lateral mass screws patients, none had radiographic complications and 35,7\% of the interspinous wire patients had complications being the segmental kyphosis the most frequent of them. Conclusions: The cervical spine injuries that underwent lateral mass screw fixation showed better radiographic results, with less complications than the interspinous wire fixation.
\end{abstract}

Keywords: Cenvical vertebrae; Fracture Fixation; Orthopedic fixation devices; Internal fixators; Spinal fractures; Spinal injuries; Cervical cerclage.

\section{RESUMEN}

Objetivo: Evaluación de la evolución radiológica de la lesión de la columna cervical tratada con fijación posterior aislada. Métodos: De 2000 a 2008, se seleccionaron 23 pacientes que cumplían los criterios de inclusión del estudio. 91,3\% eran varones y la edad media fue de 34 años y cuatro meses. El período de seguimiento promedio fue 82 meses. Se evaluó, en los exámenes de imágenes antes de la cirugía, inmediatamente después de la operación y después de seis meses de evolución, el tipo de implante, la consolidación de la artrodesis, si había aflojamiento del implante, la pérdida de la reducción, la cifosis segmentaria, la degeneración de nivel adyacente y la pseudoartrosis. Resultados: En comparación con el método de síntesis, 60,8\% de los pacientes se sometieron a fijación por cableado interespinoso, 26\% a la placa con tornillos de masa lateral y $13 \%$ a la barra con tornillos de masa lateral. De los pacientes sometidos a fijación con tornillos, ninguno presentó complicaciones radiográficas y 35,7\% de los pacientes sometidos a la fusión con el cableado interespinoso presentaron complicaciones, siendo la cifosis segmentaria la más frecuente. Conclusión: Las lesiones de columna cervical sometidas a la fusión con el tornillo de masa lateral presentaron una evolución radiográfica mejor que las de quienesfueron sometidos a fijación con cableado interespinoso, esta última presentó una mayor incidencia de complicaciones en la artrodesis.

Descriptores: Vértebras cervicales; Fijación de fractura; Dispositivos de fijación ortopédica; Fijadores internos; Fracturas de la columna vertebral; Traumatismos vertebrales; Cerclaje cervical.

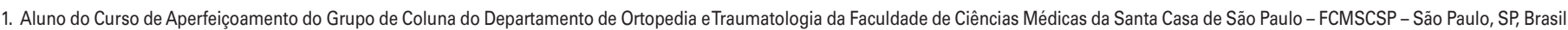
2. Pós-Graduando do Grupo de Coluna do Departamento de Ortopedia eTraumatologia da Faculdade de Ciências Médicas da Santa Casa de São Paulo - FCMSCSP - São Paulo, SP, Brasil.

3. Assistente e Professora Instrutora do Grupo de Coluna do Departamento de Ortopedia eTraumatologia da Irmandade Santa Casa de Misericórdia de São Paulo - ISCMSP - São Paulo (SP), Brasil.

4. Chefe do Grupo de Coluna e Professor-assistente do Departamento de Ortopedia eTraumatologia da Irmandade Santa Casa de Misericórdia de São Paulo - ISCMSP - São Paulo, SP, Brasil.

5. Consultor do Grupo de Coluna do Departamento de Ortopedia eTraumatologia da Faculdade de Ciências Médicas da Santa Casa de São Paulo - FCMSCSP - São Paulo, SP, Brasil.

6. Professor Adjunto do Departamento de Ortopedia eTraumatologia da Faculdade de Ciências Médicas da Santa Casa de São Paulo - FCMSCSP - São Paulo, SP, Brasil.

Trabalho realizado pelo Grupo de Coluna do Departamento de Ortopedia eTraumatologia da Santa Casa de Misericórdia de São Paulo.

Correspondência: Rua Dr Cesário Motta Jr, 61 - Vila Buarque - 1221020. São Paulo, SP. Brasil. nelsonan@yahoo.com; robertmeves@ @otmail.com

Recebido em 23/03/2011, aceito em 13/08/2011 


\section{INTRODUÇÃO}

As lesões da coluna cervical baixa, especialmente aquelas com déficit neurológico associado, estão entre as mais graves no paciente politraumatizado e que geram grande morbidade e pior qualidade de vida. Estão muito relacionadas ao trauma de alta energia e afetam principalmente a população jovem e ativa. $\mathrm{O}$ prognóstico de sobrevida e recuperação funcional tem melhorado, assim como há uma queda nas taxas de mortalidade por esse tipo de lesão $(21,8 \%)^{1}$. A evolução do atendimento pré-hospitalar e dos métodos diagnósticos de imagem tornaram o tratamento cirúrgico definitivo de estabilização e descompressão mais precoce e efetivo, com planejamento pré-operatório mais eficaz e, finalmente, o início da reabilitação quase que imediato². Apesar de inúmeros estudos de laboratórios e testes clínicos, não foi definido ainda uma terapêutica clínica efetiva e confiável para a lesão medular aguda $^{3}$. O tratamento farmacológico visando a proteção da medula espinhal da lesão secundária é discutível e ainda não é uma conduta absoluta aceita e seguida em muitos serviços no mundo ${ }^{4-9}$. Uma vez realizado a estabilização clínica do paciente e aplicado o protocolo padrão do ATLS ${ }^{\circledR 10}$ (Advanced Trauma Life Support), estudos de imagem como a radiografia simples, tomografia computadoriza e ressonância magnética são realizados. Com a análise das características da lesão cervical, será indicado o tratamento conservador ou cirúrgico ${ }^{11-13}$

O tratamento cirúrgico definitivo das lesões da coluna cervical pode ser realizado pela abordagem por via anterior ou posterior. Ambas, quando fornecem síntese rígida, estabilizam de maneira suficiente a coluna cervical a ponto de levar à consolidação óssea. A decisão pela via cirúrgica vai depender principalmente da característica da lesão cervical, das lesões associadas que limitam o posicionamento cirúrgico do paciente, da preferência do cirurgião e do material e estrutura disponível no hospital. Diversos estudos que comparam as duas abordagens já foram realizados ${ }^{11,12,14-17}$ além de tentativas de criar um fluxograma definitivo para a decisão da via cirúrgica $^{13}$. Já são bem estabelecidas as vantagens e desvantagens de cada tipo de abordagem. Se por um lado, o acesso anterior é uma via anatômica, de dissecção entre planos musculares e que fornece acesso aos elementos vertebrais anteriores como o corpo e o disco, por outro lado, coloca em risco estruturas neurovasculares, pode levar a disfagia e disfonia pós-operatória além de fornecer uma cicatriz menos estética. A via posterior tem dissecção mais fácil, facilita a redução anatômica das articulações facetarias em luxações vertebrais, não expõe estruturas neurovasculares importantes, apresenta bom leito de enxerto e fixação de implantes e deixa uma cicatriz mais estética.

O objetivo desse estudo foi analisar radiograficamente o resultado do tratamento cirúrgico de pacientes portadores de fraturas cervicais baixas pela abordagem isolada por via posterior.

\section{MATERIAIS E MÉTODOS}

Foi realizado um estudo retrospectivo devidamente aprovado no Comitê de Ética em Pesquisa (CEP) sob o número 121/10 no período compreendido entre os anos de 2000 e 2008 foram tratados no "Pavilhão Fernandinho Simonsen", da Santa Casa de Misericórdia de São Paulo, 107 pacientes com fratura cervical, e destas, 89 (83\%) atingiram a coluna cervical baixa, ou seja, entre as vértebras C3 e C7. Foram incluídos: pacientes com fratura e/ou luxação cervical baixa aguda (história inferior a 10 dias) submetidos ao tratamento cirúrgico por via posterior isolada com seguimento pós-operatório de pelo menos seis meses. Foram excluídos os pacientes com fratura ou luxação cervical de tratamento conservador ou que foram operados com fixação anterior ou combinada anterior e posterior, assim como aqueles que tinham seguimento pós-operatório inferior a seis meses ou que tiveram seus exames radiográficos extraviados. Atenderam a estes critérios 25 pacientes $(23,4 \%$ das fraturas cervicais atendidas) e houve uma perda de seguimento de dois pacientes (8\%) resultando em 23 pacientes analisados. 21 (91,3\%) destes pacientes eram do sexo masculino e dois $(8,7 \%)$ feminino com idade variando entre 16 e 56 anos (média de 34,3 anos). Foram revisados os prontuários, as radiografias e os filmes de tomografia computadorizada dos arquivos do Serviço de Arquivo Médico (SAME) da Irmandade da Santa Casa de Misericórdia de São Paulo (ISCMSP) destes pacientes. Os prontuários estavam documentados com radiografias simples da coluna nas incidências anteroposterior e perfil e filmes de tomografia computadorizada com cortes axiais e sagitais. Como rotina, todos os exames de imagem pós-operatórios do paciente são arquivados no retorno ambulatorial.

O diagnóstico foi feito através da observação da lesão óssea na coluna cervical em radiografia simples em incidências frente e perfil todas com a inclusão da transição cervico-torácica de C7-T1 no exame. Como método de classificação radiográfica da lesão foi utilizado o sistema proposto em 1982 por Allen et al. ${ }^{18}$ que diferenciam o tipo de fratura pelo mecanismo de lesão. Os seis tipos classificados são: flexão-compressão, flexão-distração, extensão-compressão, extensão-distração, compressão vertical e flexão lateral.

O déficit neurológico causado pela lesão medular foi graduado no momento do trauma de acordo com a escala de Frankel ${ }^{19}$, que se divide em cinco níveis de acordo com o grau de força muscular e a preservação da sensibilidade abaixo do nível da lesão (Quadro 1). A maior gravidade é definida pela classe A, onde há ausência de função neurológica nos níveis abaixo da lesão, e menor gravidade sendo classe $E$, onde não há déficits de força motora ou sensibilidade.

Pacientes com luxação isolada ou associada à fratura da coluna cervical tiveram a redução da lesão realizada por método indireto através de tração com halo craniano ou diretamente por redução cirúrgica pelo acesso posterior quando no insucesso do primeiro método ou na abordagem cirúrgica precoce sem a necessidade do halo craniano.

A técnica cirúrgica e o tipo do implante escolhido para cada paciente sempre foi decidida em conjunto em reunião rotineira da equipe de cirurgia da coluna do departamento. Após indicado fixação posterior isolada, o critério de escolha do implante seguiu a evolução natural destes durante todo o período analisado, ou seja, quando disponível no hospital, os implantes mais modernos sempre eram utilizados com prioridade em relação aos outros. Como método de síntese foram utilizados amarrilho interespinhoso com fio de aço (Figura 1A), placa com parafusos de massa lateral (Figura 1B) e barra com parafusos de massa lateral (Figura 1C) (Cervifix ${ }^{\circledR}$, Synthes, Paoli, Estados Unidos). Independente do método de fixação, todos os pacientes tiveram enxerto ósseo autólogo proveniente da crista ilíaca e do próprio sítio cirúrgico adicionados à área de artrodese assim como todos utilizaram colar cervical simples como imobilização pós-operatória.

O resultado pós-operatório foi avaliado de acordo com os exames de imagem em radiografia feitos imediatamente após a cirurgia e pelos realizados no seguimento ambulatorial. Foi levado em consideração na análise radiográfica o método de síntese utilizado, a presença de consolidação da artrodese após seis meses de cirurgia, a presença de soltura do implante ou perda de redução, número de níveis envolvidos na artrodese, formação de pseudartrose, degeneração óssea adjacente e formação de cifose segmentar cervical no nível da lesão. Como critério de consolidação foi adotado a presença de enxerto ósseo integrado na radiografia frente e perfil da coluna cervical com no mínimo seis meses de pós-operatório ${ }^{20}$. A cifose segmentar, quando presente, foi analisada pela medida do ângulo de Cobb nas vértebras inclusas na artrodese em radiografia com incidência lateral da coluna cervical ${ }^{21} \mathrm{com}$ pelo menos seis meses de pós-operatório.

Quadro 1. Escala de Frankel para déficit neurológico no Trauma Raquimedular.

\begin{tabular}{|c|c|}
\hline A & Ausência de força motora e sensibilidade \\
\hline B & Ausência de força motora, sensibilidade presente \\
\hline C & Força motora não útil, sensibilidade presente \\
\hline D & Força motora útil, sensibilidade presente \\
\hline E & Força motora e sensibilidade preservadas \\
\hline
\end{tabular}






Figura 1. (A) Amarria interespinhosa. (B) Placa e parafuso de massa lateral. (C) Barra e parafuso de massa lateral.

\section{RESULTADOS}

Todos os resultados estão descritos na Tabela 1.

Nesta série de 23 pacientes analisados, o tempo de seguimento dos pacientes variou entre 33 e 120 meses (média de 82,1 meses). O mecanismo de trauma mais comum foi o acidente automobilístico, seja por carro ou motocicleta, com 11 casos $(47,8 \%)$, seguido pela queda de altura com sete casos $(30,4 \%)$, atropelamento com três casos (13\%) e mergulho em água rasa em dois casos (8,7\%).

O nível de artrodese por via posterior mais realizado foi C4-C5 com seis casos (26\%), seguido por C6-C7 com cinco casos $(21,7 \%)$, C7-T1 com três casos (13\%), C5-C6 com dois (8,7\%) e C3-C4 e C2C3 com um caso $(4,3 \%)$ cada um. Houve cinco casos $(21,7 \%)$ com dois níveis de artrodese, todos entre o segmento C4-C7.

O déficit neurológico no momento do trauma foi avaliado pela escala de Frankel ${ }^{19}$ graduada em cinco níveis, sendo classe $A$ ausência de função neurológica nos níveis abaixo da lesão e classe E sem déficits de sensibilidade ou de força motora. Encontramos 12 pacientes $(52,1 \%)$ com classe $E$ e quatro $(17,4 \%)$ classe $A$. Os demais se distribuíram nas classes $\mathrm{D}, \mathrm{C}$ e B com quatro, dois e um paciente respectivamente.
Ao classificar as fraturas pelo provável mecanismo de trauma proposto por Allen e Fergusson ${ }^{18}$, a maioria dos casos segue o padrão do tipo Flexão-Distração com 14 pacientes (60,8\%), seguido pelo tipo Extensão-Compressão com sete casos (30,4\%) e Flexão-Compressão e Extensão-Distração com um paciente cada. Não houve, na série de casos, pacientes com fraturas do tipo compressão vertical ou flexão lateral.

Como método de síntese foram utilizados amarrilho interespinhoso com fio de aço (Figura 1A) em 14 (60,8\%) pacientes, placa com parafusos de massa lateral (Figura 1B) em seis (26\%) e barra com parafusos de massa lateral (Figura 1C) em três (13\%) $\left(\right.$ Cervifix $^{\circledR}$, Synthes, Paoli, Estados Unidos).

Quando analisado o resultado final, 22 dos 23 casos (95,6\%) apresentaram consolidação da artrodese, porém três desses evoluíram para cifose segmentar no local da artrodese eumpaciente evoluiu com degeneração adjacente proximal ao nível da artrodese. Houve um caso (paciente 17) que resultou em pseudartrose e cifose segmentar neste mesmo nível (Figura 2), pertencente à série de pacientes submetidos à técnica de amarrilho interespinhoso. A mesma foi documentada por meio de radiografias dinâmicas em flexão e extensão máximas em perfil da coluna cervical no pós-operatório de 12 meses onde ficou evidente a mobilidade do segmento abordado.

Os quatro pacientes que apresentaram cifose segmentar foram submetidos à técnica de artrodese posterior com amarrilho interespinhoso. A medida do ângulo de Cobb para quantificar a cifose segmentar variou de $15^{\circ}$ (Figura 3 ) até $22^{\circ}$, com média de $18,75^{\circ}$. Desses quatro pacientes, dois tinham dois níveis de artrodese e dois apenas um nível.

Todas as complicações radiográficas observadas ocorreram nos pacientes submetidos à artrodese cervical posterior pela técnica de amarrilho interespinhoso, ou seja, cinco pacientes dos $14(35,7 \%)$, sendo a mais frequente a consolidação da artrodese em cifose segmentar. Todos os pacientes submetidos à artrodese com placa ou barra fixados com parafusos de massa lateral resultaram em consolidação da artrodese sem desvio do eixo cervical sagital.

Tabela 1. Síntese dos pacientes estudados

\begin{tabular}{|c|c|c|c|c|c|c|c|c|c|}
\hline Paciente & Sexo & Idade & MT & TS & NA & Allen-Ferguson & M síntese & Frankel & Resultado \\
\hline 1 & M & 56 & auto & 35 & $\mathrm{C} 6-\mathrm{C} 7$ & Flexo-Distração & $B+P$ & A & consolidação \\
\hline 2 & M & 27 & auto & 89 & C4-C5-C6 & Extensão-Compressão & Amarrilho & $\mathrm{D}$ & consolidaçãocom cifose segmentar $30^{\circ}$ \\
\hline 3 & $\mathrm{M}$ & 51 & qa & 89 & $\mathrm{C} 2-\mathrm{C} 3$ & Extensão-Compressão & Amarrilho & $\mathrm{D}$ & consolidação \\
\hline 5 & $\mathrm{M}$ & 39 & auto & 120 & $\mathrm{C} 6-\mathrm{C} 7$ & Flexo-Distração & Amarrilho & A & consolidação \\
\hline 6 & $\mathrm{~F}$ & 52 & auto & 115 & $\mathrm{C} 5-\mathrm{C} 6$ & Flexo-Compressão & Amarrilho & $E$ & consolidação \\
\hline 7 & $\mathrm{M}$ & 28 & auto & 119 & C7-T1 & Flexo-Distração & Amarrilho & $\mathrm{D}$ & consolidação, degeneração adjacente \\
\hline 9 & $M$ & 21 & auto & 104 & $\mathrm{C} 6-\mathrm{C} 7$ & Flexo-Distração & Amarrilho & $E$ & consolidação \\
\hline 10 & $\mathrm{M}$ & 44 & atrop & 98 & $\mathrm{C4}-\mathrm{C5}$ & Extensão-Distração & $\mathrm{P}+\mathrm{P}$ & $\mathrm{B}$ & consolidação \\
\hline 11 & $\mathrm{M}$ & 27 & mar & 100 & C4-C5 & Flexo-Distração & $\mathrm{P}+\mathrm{P}$ & $E$ & consolidação \\
\hline 12 & $\mathrm{M}$ & 18 & auto & 97 & C4-C5 & Flexo-Distração & Amarrilho & $\mathrm{E}$ & consolidação \\
\hline 13 & $\mathrm{M}$ & 22 & auto & 96 & C4-C5-C6 & Extensão-Compressão & $\mathrm{P}+\mathrm{P}$ & A & consolidação \\
\hline 14 & $M$ & 17 & auto & 89 & $\mathrm{C6} 6 \mathrm{C} 7$ & Flexo-Distração & Amarrilho & $E$ & consolidação \\
\hline 18 & $\mathrm{M}$ & 50 & qa & 74 & C7-T1 & Extensão-Compressão & Amarrilho & $\mathrm{D}$ & consolidação com cifose segmentar $22^{\circ}$ \\
\hline 19 & $\mathrm{M}$ & 39 & qa & 59 & C6-C7 & Flexo-Distração & Amarrilho & A & consolidação \\
\hline 20 & $\mathrm{M}$ & 37 & qa & 63 & C4-C5 & Extensão-Compressão & $\mathrm{P}+\mathrm{P}$ & $C$ & consolidação \\
\hline 21 & $\mathrm{M}$ & 40 & atrop & 52 & C7-T1 & Extensão-Compressão & $\mathrm{P}+\mathrm{P}$ & $\mathrm{E}$ & consolidação \\
\hline 22 & $\mathrm{M}$ & 27 & auto & 33 & C4-C5 & Flexo-Distração & $\mathrm{B}+\mathrm{P}$ & $\mathrm{E}$ & consolidação \\
\hline 23 & $M$ & 20 & qa & 36 & C3-C4-C5 & Flexo-Distração & $B+P$ & C & consolidação \\
\hline
\end{tabular}

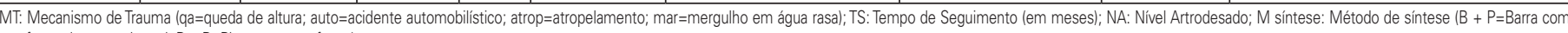
parafusos de massa lateral; $\mathrm{P}+\mathrm{P}=\mathrm{Placa}$ com parafusos) 


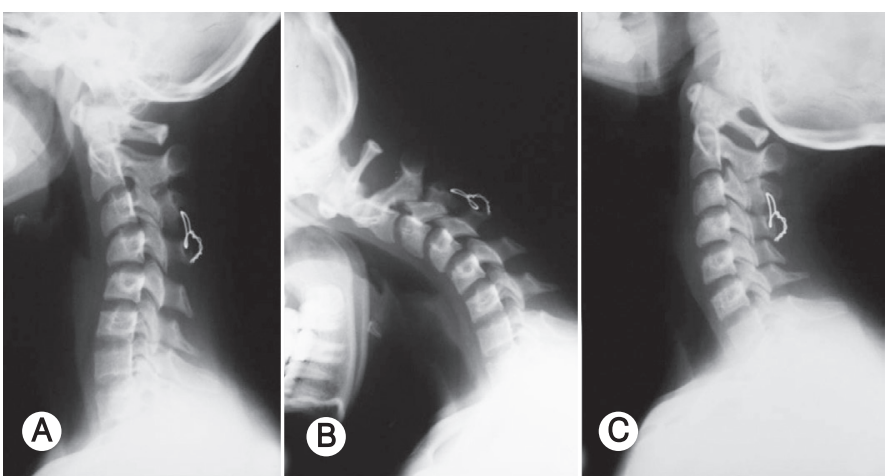

Figura 2. Pesudartrose e cifose segmentar após tentativa de artrode com amarrilho com fio de aço. Radiografia em perfil dinâmico demonstra a mobilidade do nível abordado. (A) Neutro. (B) Flexão. (C) Extensão.

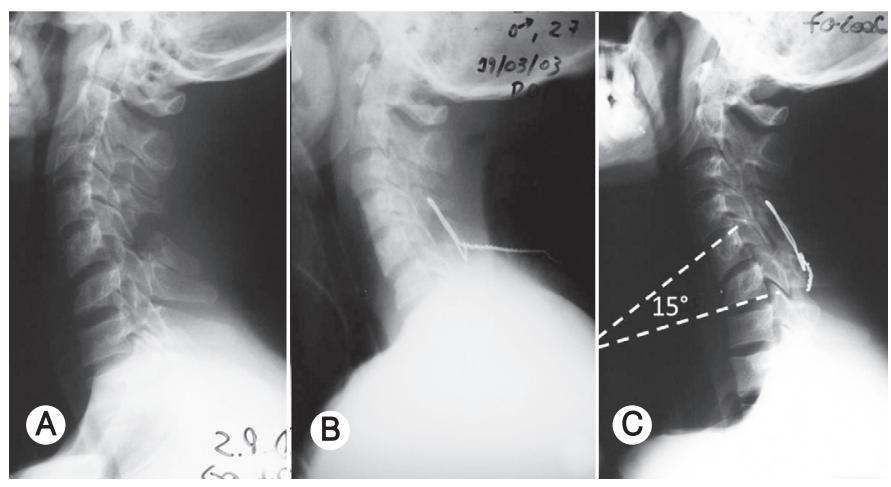

Figura 3. Exemplo de cifose segmentar. (A) Pré-operatório. (B) Pós-operatório imediato com recuperação da lordose cervical. (C) Pós-operatório seis meses com formação de cifose segmentar do nível artrodesado.

\section{DISCUSSÃO}

O tratamento cirúrgico das lesões da coluna cervical subaxial apresenta uma ampla variedade de opções para o cirurgião e ainda não existe um protocolo definitivo e absoluto que guie sua escolha pelo tipo de abordagem cirúrgica e método de fixação. O entendimento da anatomia, biomecânica e da classificação da lesão cervical subaxial é importante, assim como a história clínica, o exame físico e a análise das imagens radiográficas do paciente. A partir destes dados podemos decidir se o paciente deve ser submetido ao tratamento cirúrgico ou conservador. Em geral, há três fatores a ser considerados nesta decisão ${ }^{22}$ : instabilidade mecânica que necessita de estabilização interna; se há lesão neurológica que necessita de descompressão; e, se há outros fatores ou comorbidades do paciente que influenciem na sua decisão terapêutica. Não existe uma abordagem unificada do tratamento cirúrgico da lesão da coluna vertebral cervical.

Historicamente, a abordagem cirúrgica da lesão da coluna cervical subaxial iniciou-se pela via posterior, inicialmente por Hadra ${ }^{23}$ em 1891, seguido por Rogers ${ }^{24}$ em 1942, que propôs o método de amarrilho interespinhoso com fio de aço, método de síntese mais utilizado na nossa casuística (60,8\%). A evolução no desenvolvimento e fabricação de novos materiais e tipos de implantes introduziu as placas e parafusos na fixação posterior da coluna cervical, proposta inicialmente por Roy-Camille e Saillant ${ }^{25}$ em 1979, as quais, segundo os autores, traria maior estabilidade à artrodese vertebral. Novas técnicas cirúrgicas foram propostas, em destaque a fixação com parafusos na massa articular lateral da vértebra, proposto por Magerl et $a^{2} .^{26}$, e posteriormente sua conexão a sistemas de barras, que é o método de fixação da artrodese por via posterior mais utilizado atualmente. Considerando o ligeiro atraso que os países em desenvolvimento têm para receber e utilizar os implantes mais atuais à disposição nos países considerados desenvolvidos, a técnica cirúrgica apresentada neste serviço seguiu a evolução padrão mundial, visto que nos pacientes operados no começo desta década, consequentemente com maior tempo de seguimento pós-operatório, há uma predominância do uso do amarrilho interespinhoso. Os pacientes operados há seis anos tem na sua maioria a placa com parafusos de massa lateral como método de fixação e todos os pacientes submetidos à fixação cervical por via posterior isolada nos últimos três anos receberam a barra com parafusos de massa lateral. Estes implantes mais modernos agregam maiorestabilidade rotacional à fixação da artrodese em relação ao amarrilho interespinhoso e, consequentemente, diminuem sua taxa de falha, o que poderia levar à pseudartrose, soltura e perda do alinhamento sagital20,27-29

Não foram aplicados testes estatísticos nos resultados obtidos porque estes não apresentaram número significativo que validaria a comparação. Ao analisarmos os resultados dos métodos de fixação posterior isolados deste estudo, as fixações com parafusos de massa lateral, sejam conectados por meio de placas ou barras, não apresentaram nenhuma complicação com evidência radiográfica, ou seja, todos os casos evoluíram para consolidação da artrodese em pelo menos seis meses e sem perda do alinhamento sagital. Embora não seja estatisticamente significativo, este dado pode sugerir uma estabilidade superior e um índice de consolidação melhor do que a fixação com amarrilho interespinhoso, já que as únicas complicações radiográficas do estudo se apresentaram nos pacientes submetidos a esta última técnica.

Quando analisamos isoladamente os pacientes submetidos à técnica de amarrilho interespinhoso, notamos um índice de 35,7\% de complicações identificadas radiograficamente. A degeneração adjacente proximal observada em um único caso pode ser explicada pelo maior tempo de seguimento pós-operatório dos pacientes dessa série, já que a doença degenerativa adjacente está associada à maior exigência funcional no nível proximal ou distal à artrodese e ao tempo de evolução pós-artrodese. Um caso dessa série não apresentou sinais de consolidação da artrodese, confirmada em radiografias cervicais laterais dinâmicas em extensão e flexão máximas realizadas após seis meses de cirurgia (Figura 2) onde foi notado mobilidade no segmento artrodesado, configurando uma pseudartrose neste nível. Neste mesmo paciente, o nível abordado apresentou cifose segmentar na radiografia cervical lateral, que se corrigia na radiografia em extensão do pescoço, provavelmente pela instabilidade gerada pela pseudartose que ali se formou. Este caso, adicionado a mais três casos dessa série foram os únicos de todos os pacientes que apresentaram cifose segmentar. Façanha et al. ${ }^{30}$ analisaram 19 pacientes submetidos a artrodese da coluna cervical posterior apenas com amarrilho interespinhoso e encontraram 100\% de consolidação e 15,8\% de cifose segmentar, os quais não apresentavam alteração funcional ou dor na avaliação física.

Todos os casos submetidos à artrodese de dois níveis pela técnica de amarrilho interespinhoso, mesmo com um bom alinhamento sagital na radiografia do pós-operatório imediato, apresentaram, após seis meses, cifose segmentar no nível artrodesado, o que sugere que artrodeses com mais de um nível na coluna cervical estão mais sujeitas a perda do alinhamento sagital localizado quando feitas pela técnica de amarrilho interespinhoso. Este dado não indica um resultado funcional ruim ou uma avaliação clínica dolorosa, já que nossa avaliação é apenas radiográfica.

A análise radiográfica dos três métodos de fixação posterior para artrodese cervical após lesão traumática apontou falhas apenas no amarrilho interespinhoso. São complicações que provavelmente estão relacionadas ao menor grau de estabilidade obtido com este tipo de implante, mas que não são estatisticamente significantes. A facilidade técnica e o baixo custo do fio de aço tornam o amarrilho interespinhoso bastante atrativo em comparação com os parafusos de massa lateral, placas e barras, o que explica seu uso ainda freqüente, principalmente nos países menos desenvolvidos. Apesar de ter apresentado uma incidência de complicações maior, a fixação com amarrilho interespinhoso ainda é um método válido e que atende adequadamente os serviços de trauma com poucos recursos. 


\section{CONCLUSÃO}

A abordagem cirúrgica da lesão da coluna vertebral cervical pela via posterior isolada é uma boa opção quando bem indicada. Os resultados da análise radiográfica deste tipo de tratamento mostraram uma tendência favorável à artrodese com parafusos de massa lateral em relação à técnica com amarrilho, porém são necessários estudos com uma amostra maior de pacientes para tornar essa afirmação válida. A escolha do tipo de implante deve priorizar, quando disponível, os parafusos com fixação na massa lateral da vértebra, porém, a fixação com amarrilho interespinhoso continua sendo uma opção válida e de baixo custo.

\section{REFERÊNCIAS}

1. Oliveira AR, Avanzi O. Estudo sobre a mortalidade de pacientes com fratura da coluna cervical durante o período de hospitalização. Rev Bras Ortop. 2002; 37(3):89-96.

2. Burns AS, Ditunno JF. Establishing prognosis and maximizing functional outcomes after spinal cord injury: a review of current and future directions in rehabilitation management. Spine (Phila Pa 1976). 2001;26(Suppl 24):S137-45.

3. Gupta R, Bathen ME, Smith JS, Levi AD, Bhatia NN, Steward O. Advances in the management of spinal cord injury. J Am Acad Orthop Surg. 2010:18(4):210-22.

4. Bracken MB, Shepard MJ, Holford TR, Leo-Summers L, Aldrich EF, Fazl M, et al. Methylprednisolone or tirilazad mesylate administration after acute spinal cord injury: 1-year follow up. Results of the third National Acute Spinal Cord Injury randomized controlled trial. J Neurosurg. 1998;89(5):699-706

5. Fehlings MG. Editorial: recommendations regarding the use of methylprednisolone in acute spinal cord injury: making sense out of the controversy. Spine (Phila Pa 1976). 2001:26(Suppl 24):S56-7.

6. Bracken MB, Holford TR. Effects of timing of methylprednisolone or naloxone administration on recovery of segmental and long-tract neurological function in NASCIS 2. J Neurosurg. 1993:79(4):500-7.

7. Bracken MB, Shepard MJ, Holford TR, Leo-Summers L, Aldrich EF, Fazl M, et al. Administration of methylprednisolone for 24 or 48 hours or tirilazad mesylate for 48 hours in the treatment of acute spinal cord injury. Results of the Third National Acute Spinal Cord Injury Randomized Controlled Trial. National Acute Spinal Cord Injury Study. JAMA. 1997;277(20):1597-604

8. Hugenholtz H, Cass DE, Dvorak MF, Fewer DH, Fox RJ, Izukawa DM, et al. High-dose methylprednisolone for acute closed spinal cord injury-only a treatment option. Can J Neurol Sci. 2002;29(3):227-35.

9. Ackery A, Tator $C$, Krassioukov A. A global perspective on spinal cord injury epidemiology. J Neurotrauma. 2004:21(10):1355-70.

10. American College of Surgeons. ATLS: Advanced Trauma Life Support Program for Doctors. 7th ed. Chicago:American College of Surgeons; 2008.

11. Chapman JR, Anderson PA. Internal fixation techniques for the treatment of lower cervical spine injuries. J Int Orthop Trauma. 1991(1):205-19.

12. Raynor RB. Anterior or posterior approach to the cervical spine: an anatomical and radiographic evaluation and comparison. Neurosurgery. 1983;12(1):7-13.

13. Dvorak MF, Fisher CG, Fehlings MG, Rampersaud YR, Oner FC, Aarabi B, et al. The surgical approach to subaxial cervical spine injuries: an evidence-based algorithm based on the SLIC classification system. Spine (Phila Pa 1976). 2007:32(23):2620-9.

14. Sutterlin CE 3rd, McAfee PC, Warden KE, Rey RM Jr, Farey ID. A biomechanical evaluation of cervical spinal stabilization methods in a bovine model. Static and cyclical loading. Spine (Phila Pa 1976). 1988;13(7):795-802.

15. Coe JD, Warden KE, Sutterlin CE 3rd, McAfee PC. Biomechanical evaluation of cervical spinal stabilization methods in a human cadaveric model. Spine (Phila Pa 1976). 1989;14(10):1122-31.
16. Brodke DS, Anderson PA, Newell DW, Grady MS, Chapman JR. Comparison of anterior and posterior approaches in cervical spinal cord injuries. J Spinal Disord Tech. 2003:16(3):229-35

17. Do Koh Y, Lim TH, Won You J, Eck J, An HS. A biomechanical comparison of modern anterior and posterior plate fixation of the cervical spine. Spine (Phila Pa 1976). 2001;26(1):15-21.

18. Allen $B L \mathrm{Jr}$, Ferguson $\mathrm{RL}$, Lehmann TR, O'Brien RP. A mechanistic classification of closed, indirect fractures and dislocations of the lower cervical spine. Spine (Phila Pa 1976). $1982 ; 7(1): 1-27$.

19. Frankel HL, Coll JR, Charlifue SW, Whiteneck GG, Gardner BP, Jamous MA, et al. Long-term survival in spinal cord injury: a fifty year investigation. Spinal Cord. $1998 ; 36(4): 266-74$

20. Anderson PA, Henley MB, Grady MS, Montesano PX, Winn HR. Posterior cervical arthrodesis with AO reconstruction plates and bone graft. Spine (Phila Pa 1976) 1991:16(Suppl 3):S72-9.

21. Harrison DE, Harrison DD, Cailliet R, Troyanovich SJ, Janik TJ, Holland B. Cobb method or Harrison posterior tangent method: which to choose for lateral cervical radiographic analysis. Spine (Phila Pa 1976). 2000;25(16):2072-8.

22. Kwon BK, Vaccaro AR, Grauer JN, Fisher CG, Dvorak MF. Subaxial cervical spine trauma. J Am Acad Orthop Surg. 2006;14(2):78-89.

23. Hadra BE. Wiring the spinous processes in Pott's disease. J Bone Joint Surg Am 1891; 1 1-4:206-10

24. Rogers WA. Treatment of fracture-dislocation of the cervical spine. J Bone Joint Surg. 1942:24:245-58

25. Roy-Camille R, Saillant G. Chirurgie du rachis cervical inferieur. Techniques d'osteosynthesedur rachis cervical inferior. In: Premieres Journées de la Pitie, Paris, Masson; 1979. p. 36-50

26. Magerl F, Grob D, Seemann D. Stable dorsal function of the cervical spine using Hookplates. In: Kehr P, Weidner A, edtors. Cervical spine I. Berlin:Springer-Verlag; 1987. p. 217-21.

27. Nazarian SM, Louis RP. Posterior internal fixation with screw plates in traumatic lesions of the cervical spine. Spine (Phila Pa 1976). 1991;16(Suppl 3):S64-71.

28. Shapiro S, Snyder W, Kaufman K, Abel T. Outcome of 51 cases of unilateral locked cervical facets: interspinous braided cable for lateral mass plate fusion compared with interspinous wire and facet wiring with iliac crest. $J$ Neurosurg.1999;91(Suppl 1):19-24

29. Cooper PR, Cohen A, Rosiello A, Koslow M. Posterior stabilization of cervical spine fractures and subluxations using plates and screws. Neurosurgery. 1988;23(3):300-6.

30. Façanha FAM, Kusabara R, Avanzi O. A amarria interespinhosa como síntese nas fraturas da coluna cervical de C3-T1. Rev Bras Ortop. 1994;29(6):401-10 